\title{
Determinantes ambientales y desafíos para el ordenamiento forestal sustentable en los algarrobales del Monte, Argentina
}

\author{
Pablo E. Villagra ${ }^{1,2}$ 四 \& Juan A. Alvarez ${ }^{1 / 2}$ \\ ${ }^{1}$ Instituto Argentino de Nivología, Glaciología y Ciencias Ambientales. CONICET Mendoza, Argentina. ${ }^{2}$ Facultad de Ciencias \\ Agrarias. Universidad Nacional de Cuyo. Mendoza. Argentina.
}

\begin{abstract}
RESUMEN. Una proporción sustancial de los bosques en retroceso se ubican en zonas áridas. Los algarrobales de la Provincia Biogeográfica del Monte se encuentran entre los bosques con mayores limitaciones ambientales del país, lo que hace que la idea de un ordenamiento forestal sustentable sea difícil de lograr. En este trabajo nos proponemos discutir los determinantes ambientales y de historia de uso que limitan su potencialidad productiva, las estrategias territoriales y de manejo que contribuyan a aumentar la sustentabilidad de su aprovechamiento, y la conservación y la recuperación de los bosques degradados. Los algarrobales presentan un gradiente latitudinal en la estructura poblacional; esto determina su potencial forestal. Los bosques del sector norte contienen árboles de mayor tamaño, crecimiento y productividad, lo que permite pensar en un manejo forestal maderero. Por su parte, en el centro y en el sur sólo se podrían extraer productos de menor valor maderero (i.e., leña y postes), junto con manejos en los que la ganadería extensiva y otros productos no madereros sean la actividad principal. La precipitación es el mayor determinante de la receptividad ganadera, mientras que la historia de uso generó distintos estados de degradación en los bosques. El gran desafío es poder realizar un ordenamiento territorial que considere la heterogeneidad espacial descripta y que mejore los procesos tecnológicos usados, de forma de optimizar el aprovechamiento, y que permita decidir las actividades prioritarias para cada área. Se propone la comparación analítica de mapas de distribución potencial, histórica y actual como una herramienta con fuertes implicancias desde el punto de vista del manejo y la conservación de los bosques, ya que permite discriminar zonas de distintos potenciales productivos, zonas potenciales de reforestación y zonas sin vocación forestal.
\end{abstract}

[Palabras clave: Prosopis, sustentabilidad, manejo forestal, bosques de zonas áridas, silvicultura, ordenamiento territorial, OTBN]

\begin{abstract}
Aвstract. Environmental determinants and challenges for sustainable forest management in the Prosopis flexuosa woodlands from the Monte desert, Argentina. A significant proportion of the world-degraded forests is located in arid zones. The $P$. exuosa woodlands from the Monte Biogeographic Province are among the most environmentally constrained forest in the country, which suggests the sustainable forest management is difficult to achieve. In this paper, we propose to discuss the environmental determinants of woodland productivity, and to evaluate territorial and management strategies that contribute to increase the sustainability of their use, and to the conservation and restoration of degraded forests. These woodlands show a latitudinal gradient in the population structure; this determines their forestry potential. The woodlands from the Northern sector have trees of greater size and higher growth and productivity, allowing us to think about timber forest management. In the central and southern sectors, in turn, it would only be possible to extract products of lower economic value (i.e., firewood and posts), integrated with extensive livestock and other non-timber products use. Precipitation is the main determinant of livestock carrying capacity, and the history of use has generated different states of woodland degradation. The great challenge is to be able to carry out a territorial planning that takes into account the spatial heterogeneity described above, that improves the technological processes used in order to optimize woodland production, and that makes it possible to decide on the priority activities for each area. The analytical comparison of maps of potential, historical and current distribution is proposed as a tool with strong implications on the woodland management and conservation, since it allows for the discrimination of areas with forest-use potential, reforestation zones and areas with no forest vocation.
\end{abstract}

[Keywords: Prosopis, sustainability, arid zone woodlands, forestry, land use planning, OTBN]

\section{PROBLeMÁticA DE LOS BOSQUES DEL MUNDO Y DE ZONAS ÁRIDAS}

La pérdida y la degradación de los bosques nativos es uno de los problemas globales con consecuencias en el clima, en la disminución de la biodiversidad y en la provisión de servicios ecosistémicos. El 41\% de los bosques del mundo se encuentran en zonas áridas y

Editores invitados: Rosina Soler y Juan Gowda $\bar{\square}$ villagra@mendoza-conicet.gob.ar semiáridas (Bastin et al. 2017) y una proporción importante de los bosques en retroceso se encuentran en ellas. Estos bosques son claves por los valiosos servicios ambientales que brindan. Sin embargo, dado que presentan una diversidad relativamente baja y escasos recursos naturales aprovechables, a lo largo del tiempo no han llegado a captar tanta atención como otras áreas boscosas. No 
obstante, estos sistemas suministran productos y servicios que benefician de forma directa a los pobladores locales (Cuba Salerno 1998). Si se tiene en cuenta los patrones particulares de distribución de la diversidad que poseen y la variedad de amenazas a las que son sometidos, en su mayoría de origen antrópico (Miles et al. 2006), la conservación y manejo sustentable de estos bosques se convierte en una prioridad a nivel global.

Los objetivos de conservación y aprovechamiento del bosque se han contrapuesto a lo largo de la historia, pero los nuevos enfoques de manejos de bosques los integran en lo que la FAO llama ordenamiento forestal sustentable. Este enfoque implica considerar para la planificación no sólo la magnitud de los recursos forestales, sino también la diversidad, la vitalidad, las funciones productivas y protectoras, y las funciones socioeconómicas del bosque, y el marco jurídico e institucional (FAO 2007, 2016). En sentido estricto, la sustentabilidad de un manejo implica mantener tanto el capital natural como la producción de bienes y servicios que satisfagan las necesidades socioeconómicas y culturales de la población (Sarandón 2002). Si bien esta visión es muy tentadora, en bosques de zonas áridas, las bajas tasas de regeneración y productividad hacen que el desafío de lograr un manejo sustentable desde el punto de vista ambiental, económico y social sea más difícil que en otras áreas, al menos a escala local o predial. Esto lleva a pensar en la necesidad de considerar la variabilidad espacial del potencial productivo y la posibilidad de integrar manejos a una escala más amplia, incluyendo desarrollo de políticas territoriales que contribuyan con la sustentabilidad social del manejo.

Los algarrobales de la provincia biogeográfica del Monte, en el oeste árido argentino, se encuentran entre los bosques con mayores limitaciones ambientales del país. En este trabajo nos proponemos discutir los determinantes ambientales y de historia de uso que limitan su potencialidad productiva, y las estrategias territoriales y de manejo que contribuyan a aumentar la sustentabilidad de su aprovechamiento, y la conservación y recuperación de los bosques degradados.

\section{Los ALGARRobales DEL MONTE: HISTORIA DE USO}

La región del Monte ocupa el oeste árido de la Argentina, con precipitaciones medias anuales entre 30 y $350 \mathrm{~mm}$. Las temperaturas medias varían entre 13 y $18{ }^{\circ} \mathrm{C}$ (Labraga and Villalba 2009). Se caracteriza por ser un mosaico de tres tipos de asociaciones vegetales: la estepa de arbustos, dominada por especies de la familia Zigophyllaceae (Larrea spp. y Bulnesia retama), las comunidades asociadas a suelos particulares como las comunidades halófitas, psamófilas, de suelos arcillosos o riparias, y el bosque dominado por especies del género Prosopis (conocido como "algarrobal") que forman unidades boscosas de distinta magnitud en el fondo de valles y bolsones (Figura 1). Los algarrobales presentan un estrato arbóreo abierto dominado por $P$. flexuosa o P. chilensis, acompañados por Geoffroea decorticans; el estrato arbustivo es dominado por Capparis atamisquea, especies del género Larrea, Suaeda divaricata y Atriplex lampa. El estrato herbáceo está dominado por Leptochloa crinita y Pappophorum caespitosum, especies de buen valor forrajero.

Como las precipitaciones menores a 350 $\mathrm{mm} /$ año no son suficientes para el desarrollo de los algarrobos, este bosque sólo aparece en lugares con agua subterránea accesible (nivel freático entre 5-30 m) o por la presencia de cauces permanentes o temporarios (Morello 1958; Alvarez y Villagra 2009; Jobbágy et al. 2011). Esta agua extra hace que estos sitios presenten una productividad superior a lo esperado por las precipitaciones (Contreras et al. 2011), lo que los convierte en zonas con mayor potencial para el manejo forestal y ganadero.

La presencia de algarrobos facilita el establecimiento de otras especies y aumenta la fertilidad del suelo por el aporte de materia orgánica bajo su dosel (Rossi and Villagra 2003; Alvarez et al. 2009; Cesca et al. 2012). Este efecto es más marcado cuanto más árida es la zona, y se observan sitios donde los pastos forrajeros crecen exclusivamente bajo el dosel del algarrobo (Cesca et al. 2012). De esta forma, un dosel de algarrobos puede aportar forraje como fruto, mejorar la calidad de otros forrajes, proteger a otras especies de las heladas y estabilizar la producción durante sequías (Karlin and Díaz 1984; Cesca et al. 2012). Esto sugiere que para lograr la sustentabilidad se debe asegurar la presencia del estrato arbóreo, ya sea por conservación de lo existente o por restauración de las áreas degradadas.

En América del Sur, la relación del ser humano con las especies del género se remonta a épocas precolombinas, cuando constituían 


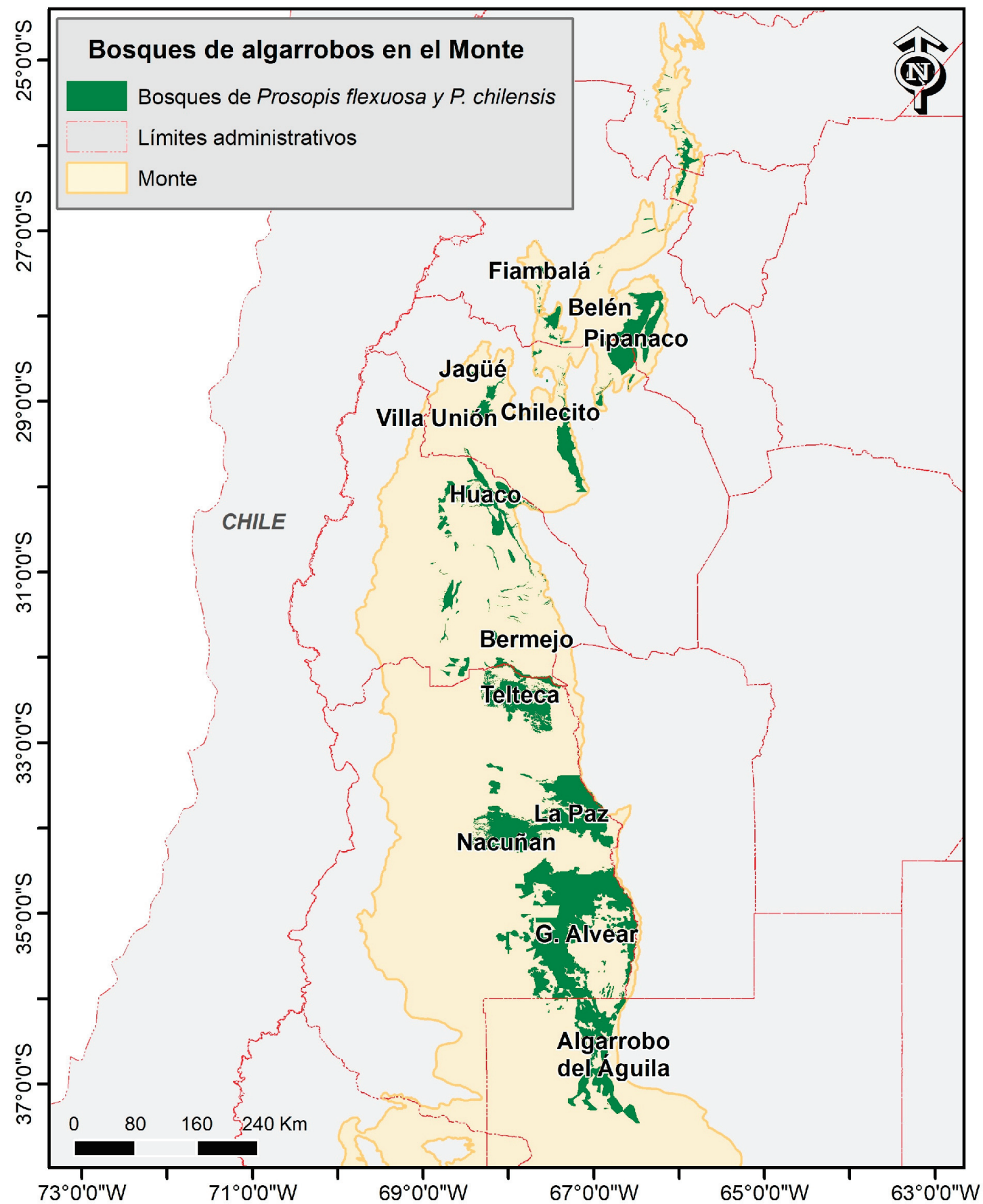

Figura 1. Principales unidades boscosas en la Provincia Biogeográfica del Monte. La distribución de los algarrobales (en verde) es una síntesis de información generada en distintos trabajos (Alvarez et al. 2006, 2015; Rojas 2013; Cesca et al. 2014). Elaboración: F. Rojas.

Figure 1. Main woodland units in the Monte desert. The distribution Prosopis woodlands (in green) is a synthesis of information generated in different works. (Alvarez et al. 2006, 2015; Miner et al. 2010; Rojas 2013; Cesca et al. 2014). Map preparation: F. Rojas.

el principal alimento de las personas y los animales. Como ejemplo se puede citar a los huarpes de Mendoza, cuyas poblaciones se establecían donde había algarrobos (Solbrig and Cantino 1975). Como los algarrobales del Monte se ubican en zonas con agua, también constituyen sitios preferidos por los asentamientos humanos; en la actualidad, 
muchos pueblos y ciudades se encuentran sobre antiguos algarrobales.

El algarrobal fue la principal comunidad vegetal que sustentó las poblaciones humanas al proveer alimento, forraje, madera, postes, sombra y otros productos. Desde fines del siglo XIX se produjo un incremento en la presión sobre estos bosques como consecuencia de la expansión del ferrocarril, lo que permitió la extracción de $P$. flexuosa para leña, carbón, y para la producción de gas pobre para el alumbrado urbano. Posteriormente, en las primeras décadas del siglo XX se produjo la mayor expansión de la vitivinicultura, con la consecuente extracción de postes para la conducción de los viñedos. Además, estos cultivos se expandieron sobre áreas donde había algarrobales. En la segunda mitad del siglo XX se produjo una mayor demanda de madera para muebles, lo que afectó principalmente a los bosques del norte del Monte (Roig 1993; Villagra et al. 2004). La actividad minera en los valles del norte usaba la leña de algarrobo como combustible para sus fundiciones (Rojas 2013). Actualmente, la economía del Monte se sustenta en dos tipos de sistemas productivos, uno de alto rendimiento en los oasis irrigados y el otro en las zonas no irrigadas utilizadas principalmente para ganadería extensiva (Prieto and Abraham 1998; Abraham and Prieto 1999; Villagra et al. 2009).

El manejo aplicado históricamente en estos bosques trajo consecuencias en los tres ejes de consideración al evaluar la sustentabilidad de un sistema: a) Eje ambiental. La erosión de los recursos naturales que provocan las actividades extractivas sostenidas en el tiempo sin considerar su tasa de regeneración. El algarrobal sufrió una retracción de su superficie y una degradación de sus parámetros estructurales, y presenta distintos grados de desertificación, en algunos casos irreversibles, al haber alterado procesos funcionales claves (Villagra et al. 2009; Cesca et al. 2014). El uso ineficiente del recurso hídrico en los oasis afecta el escurrimiento superficial $y$ el agua que reciben las zonas de secano; $b$ ) Eje económico. El uso doméstico de productos del bosque representa una parte sustancial de la economía del puesto ganadero y del ingreso anual del grupo familiar. La pérdida de productividad forestal y ganadera de los bosques, y la disminución de la demanda de productos del bosque desde los oasis hizo que se acentúen las diferencias entre las posibilidades económicas de los pobladores del desierto y los de los oasis irrigados; c) Eje socio-cultural. los cambios ecosistémicos causados por el uso del bosque y la apropiación del agua en los oasis modificaron el tipo de actividades productivas de los pobladores; esto limitó la actividad a un sistema de ganadería extensiva (Goirán et al. 2013). La escasa densidad poblacional del secano y su desvinculación histórica con los centros urbanos y agrícolas llevaron a una limitada infraestructura en servicios básicos. En la última década se comenzó a tener en cuenta y revertir esta carencia de infraestructura.

\section{Potencial PRODUCTIVO DE LAS DISTINTAS UNIDADES BOSCOSAS DEL MONTE}

Las condiciones ambientales extremas y las fuertes adaptaciones determinaron que las poblaciones de Prosopis spp. del Monte presenten rasgos estructurales muy variables. Una primera fuente de variación es la latitud; esta variación determina el potencial productivo. Al norte, con períodos de crecimiento más largos, se observan árboles de mayor tamaño y porte erecto, plantas monopódicas y tasas de crecimiento diamétrico mayores a $6 \mathrm{~mm} /$ año. En las poblaciones sureñas, los algarrobos son multifustales, de forma decumbente y con un crecimiento diamétrico que no supera $\operatorname{los} 2 \mathrm{~mm} / \mathrm{año}$. Estas diferencias determinan una variación en la productividad forestal de casi un orden de magnitud entre los bosques norteños y los sureños (Figura 2) (Villagra et al. 2004, 2005; Alvarez and Villagra 2009).

La combinación del estado sanitario y el hábito de crecimiento de los árboles determina el tipo de productos forestales. Los productos madereros cambian su nomenclatura y dimensiones según las regiones del Monte. En bosques de Catamarca y La Rioja, las características de los árboles hacen que sea posible planificar actividades de uso forestal maderero. Así, la antigua producción de tablas para parquet determinó la extracción de trozas mayores a $0.35 \mathrm{~m}$ de diámetro y largos mayores a $1.5 \mathrm{~m}$. La industria vitivinícola consumió postes, medio postes, rodrigones, estacones y parraleros para los sistemas de conducción. En la actualidad, la mayor cantidad de madera usada corresponde a leña. Por el contrario, en el centro y sur sólo sería posible la extracción de productos de menor valor maderero (i.e., 

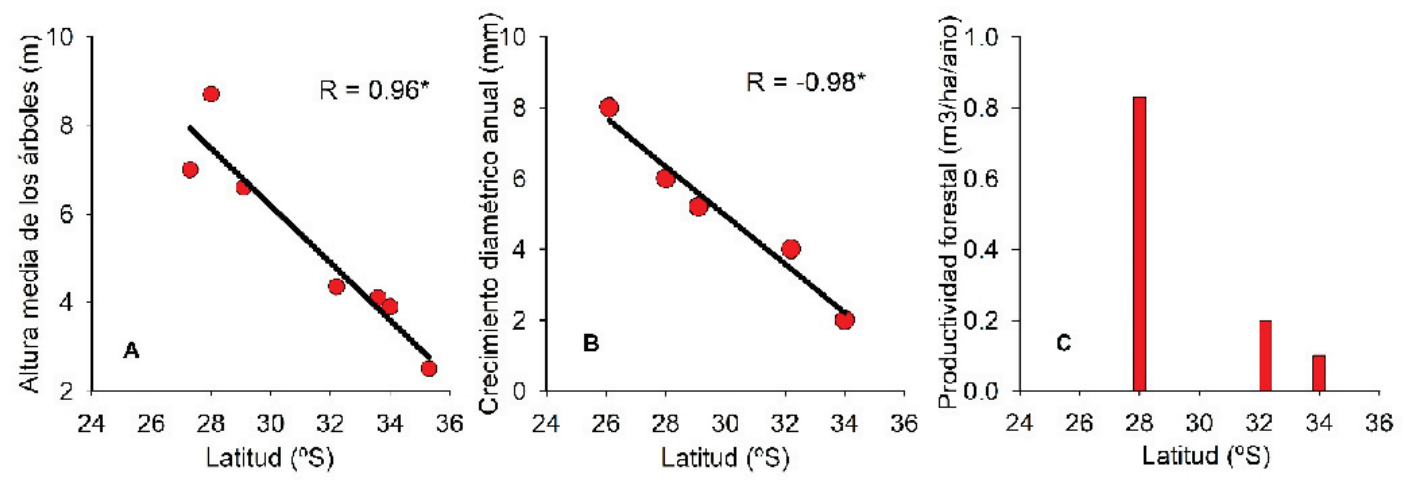

Figura 2. Cambios latitudinales en la estructura y la productividad del bosque. A) Altura media de los árboles; B) Crecimiento diamétrico anual medido en incremento del diámetro basal equivalente de sus fustes; C) Productividad forestal maderera estimado a partir de ecuaciones de regresión. Los datos provienen de Villagra et al. (2005), Alvarez et al. $(2006,2015)$ y Cesca et al. (2014), complementados con datos no publicados.

Figure 2. Latitudinal changes in woodland structure and productivity. A) Average tree height; B) Annual diameter growth measured in increment of the equivalent basal diameter of their stems; C) Estimated forest productivity using regression equations. Data from Villagra et al. (2005), Alvarez et al. (2006, 2015) and Cesca et al. (2014), supplemented by unpublished data.

leña y postes) integrada a manejos en los que la ganadería extensiva y otros productos no madereros sean la actividad principal. Por otro lado, las prácticas aplicadas pueden adecuar la estructura del bosque a los fines del manejo. En los bosques riojanos, la capacidad de rebrote luego de la poda del fuste principal permite la producción de muchos rebrotes, lo que aumenta la cantidad por hectárea de productos forestales destinados principalmente a postes. Esta posibilidad productiva depende de la altura de poda, con una mayor producción en fustes cortados entre 0.7 y $1 \mathrm{~m}$, según el conocimiento empírico de los pobladores de la zona. En los sitios más productivos, la antigua provisión de postes para la conducción de viñedos en los oasis se transformó a postes para soporte de alambrados en campos ganaderos.

Los pobladores de la región árida de la Argentina dan a los bosques un uso silvopastoril. La ganadería caprina, y en menor medida la vacuna, forma parte sustancial de su economía doméstica. La variabilidad espacial de la receptividad ganadera está determinada especialmente por las precipitaciones. Así, en zonas donde las lluvias superan los $300 \mathrm{~mm} /$ año, la receptividad ganadera es cercana a 10 ha/EV (equivalente vaca), mientras que en las zonas más áridas puede llegar a las 60 ha/EV. El efecto del consumo animal es variado en el ambiente boscoso ya que la dieta del ganado vacuno se compone de diferentes pastos y la caprina está compuesta por pastos y arbustos, según la época del año (Guevara et al. 2009). La instalación y el funcionamiento de puestos ganaderos genera gradientes ambientales producto de la interacción de los efectos de distintos disturbios sobre las condiciones edáficas, la disponibilidad de leña seca y el reclutamiento o mortalidad de distintas especies (Meglioli et al. 2014). Se propusieron técnicas de manejo con rotación que pueden aumentar la productividad y la sustentabilidad del manejo del bosque en las zonas menos áridas (Guevara et al. 2009). Mientras tanto, en las zonas más áridas se resaltó la importancia de los arbustos forrajeros y la protección de la cobertura arbórea para el manejo de los sistemas caprinos (Allegretti et al. 2005; Cesca et al. 2012).

Otros productos del bosque se pueden incluir en proyectos integrados de manejo forestal sustentable. Por ejemplo, el uso de los frutos de algarrobo como reserva de forraje o para consumo humano a través de la producción de harina para ser incorporado como insumo de múltiples productos. La harina de algarroba, que los habitantes del bosque usan tradicionalmente, fue incluida en el año 2014 en el Código Alimentario Nacional (artículo 681). También se iniciaron proyectos en los que los pobladores del bosque se organizaron para producir y vender productos que incluyan harina, con resultados prometedores (Martínez de Escobar et al. 2015). La producción de polen y néctar es un recurso importante. En menor medida se puede considerar el uso de junquillo (Sporobolus rigens) como materia prima producir escobas y cestería (Vilela et al. 2009). Otra alternativa económica es el turismo alternativo, con algunas experiencias 
conocidas en las localidades de Asunción, Lagunas del Rosario y Telteca (Lavalle, Mendoza).

\section{ENFOQUES PARA EL ORDENAMIENTO FORESTAL SUSTENTABLE.}

Uno de los problemas que tuvo la planificación del manejo de los bosques del Monte se relaciona con el hecho de haberse basado sólo en la distribución y la estructura del bosque en un momento dado, sin considerar su dinámica pasada y futura. Se aplicaron normas dasocráticas arbitrarias, que lejos de tener en cuenta la tasa de regeneración se basaron en prácticas tradicionales o extrapoladas de otras regiones. La distribución actual de los bosques depende no sólo de la

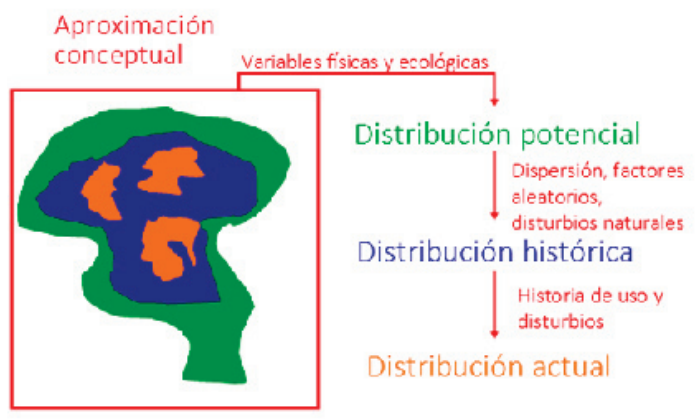

Figura 3. Aproximación conceptual para evaluar áreas con distinta potencialidad para el manejo de bosques. La zona verde representa la distribución potencial hipotética del bosque, determinada por las variables ambientales. El área azul representa la distribución histórica del bosque, modificada por la historia de uso y por disturbios, determinando la distribución y estructura actual representada por el área naranja. Las flechas indican los procesos determinantes de cada una de las distribuciones.

Figure 3. Conceptual approach to evaluate areas with different potential for forest management. The green zone represents the hypothetical potential distribution of the woodlands determined by the environmental variables. The blue area represents the historical distribution, modified by the history of use and disturbances determining the current distribution and structure represented by the orange area. The arrows indicate the determining processes of each of the distributions. presencia de condiciones ambientales que permitan su desarrollo, sino también de factores históricos de dispersión, disturbios y uso que puedan restringir su distribución, y de su capacidad de regeneración o resiliencia (Figura 3). La distribución potencial abarca el área que presenta condiciones ambientales que satisfagan los requerimientos de una especie o de una comunidad. Para el Monte, Perosa et al. (2014) desarrollaron un modelo de distribución potencial a partir de variables predictoras y puntos conocidos de presencia de bosques que permite distinguir las zonas más aptas para la presencia de bosques. Por su parte, los estudios de distribución histórica o de línea de base han sido encarados a través de fuentes documentales (Prieto and Abraham 1998; Abraham and Prieto 1999; Prieto et al. 2003; Rojas et al. 2014a). La comprensión de estos procesos tiene implicancias notables desde el punto de vista del manejo y la conservación de los bosques ya que permite discriminar zonas de distintos potenciales productivos, zonas potenciales de reforestación y zonas sin vocación forestal.

Según este enfoque, la comparación analítica de mapas de distribución potencial, histórica y actual nos permitirá planificar el uso del territorio (Figura 4). Las zonas con una alta probabilidad de ocurrencia de bosque, y donde - a pesar del uso histórico- se observa una estructura actual bien conservada, podrían ser tenidas en cuenta para planificar actividades con uso forestal maderero sustentable (Tabla 1). En algunos algarrobales de la zona norte (e.g., Bolsón de Pipanaco) encontramos bosques que se incluirían en esta situación. Por otro lado, las zonas donde las condiciones ambientales son sub-óptimas o marginales suelen presentar una baja capacidad de recuperación natural al uso. En estas áreas, si el bosque se encuentra en buenas condiciones debido al poco uso histórico, se recomienda limitar su uso con fines forestales y promover otras actividades compatibles con la conservación del bosque. El área de Telteca

Tabla 1. Metodología para identificar zonas con potencial productivo, zonas con necesidad de restauración o conservación y zonas no forestales, en función de la distribución potencial, la distribución histórica e historia de uso, y de su estructura actual.

Table 1. Identification of areas with forestry potential, areas requiring restoration or conservation and non-forest areas according to their potential distribution, historical distribution and history of use, and their current structure.

\begin{tabular}{lcccc}
\hline & \multicolumn{2}{c}{ Probabilidad potencial } & \multicolumn{2}{c}{ Distribución } \\
Zonas & He ocurrencia de bosque & histórica & uso & Estructura actual \\
\hline Zonas de potencial productivo alto & Alta & Presencia & Alto & Buen desarrollo \\
Zonas de conservación & Baja o media & Presencia & Bajo & Buen desarrollo \\
Zona de restauración & Alta o baja & Presencia & Alto & Ausencia o degradado \\
Zona no forestal & Nula o muy baja & Ausencia & Nulo & Ausencia \\
\hline
\end{tabular}


(Mendoza) presenta bosques en buen estado de conservación porque al estar entre médanos no se tuvo un acceso sencillo en la época de mayor uso de estos bosques. Además, los trabajos históricos mencionados mostraron una mayor extensión de algarrobales para mediados del siglo XIX que en la actualidad (Figura 4). En estas áreas donde se registró pérdida forestal y no está ocurriendo una recuperación pasiva, será necesario plantear estrategias de restauración ecológica que permitan restablecer procesos funcionales claves. Se evaluó la variabilidad genética de $P$. flexuosa y $P$. chilensis, y distintas técnicas de revegetación, incluyendo también otras formas de vida, con el objetivo de desarrollar tecnologías de restauración y forestación de zonas degradadas por uso y salinidad (Cony 1996; Villagra et al. 2017). Por último, lugares donde el análisis de distribución potencial arroja una probabilidad muy baja o nula de existencia y no hay registro histórico de bosques debería considerarse tierra sin vocación forestal y no implementarse planes de forestación, a menos que se piense aplicar herramientas de manejos especiales, como la aplicación de riego en el caso del Monte (Tabla $1)$. Se propone, entonces, un ordenamiento territorial que considere la heterogeneidad espacial del potencial productivo del bosque, con el finde acercarse a la sustentabilidad a escala regional (Figura 4).

\section{DESAFÍOS PARA EL MANEJO DE LOS BOSQUES DEL MONTE}

De las consideraciones propuestas por FAO (2007) para encarar el ordenamiento forestal sustentable surgen las necesidades de conocimientos y de gestión para lograr una estrategia territorial que permita mejorar aspectos productivos y económicos. Además, también se persigue optimizar la capacidad del ambiente para producir bienes y servicios. Básicamente, es necesario evaluar los factores que determinan la capacidad productiva (en sentido amplio) y la tasa de regeneración de los recursos naturales de los bosques $\mathrm{y}$, por otro lado, las necesidades y demandas de los pobladores locales para satisfacer necesidades económicas y culturales. En el caso del Monte se lograron avances en casi todos los aspectos propuestos; sin embargo, la integración de este conocimiento en verdaderos planes de ordenamiento territorial sustentable fue sólo parcial. Esto responde a que, a pesar de los avances, todavía están incompletos para la región los datos primarios correspondientes al avance y retroceso del bosque, su área total, regeneración natural y zonas
Distribución potencial

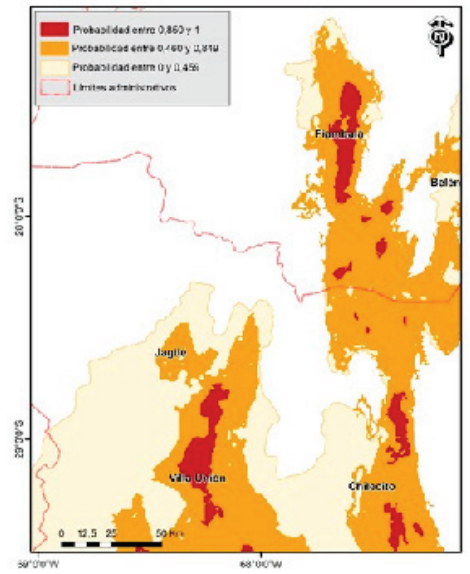

Distribución histórica (1850)

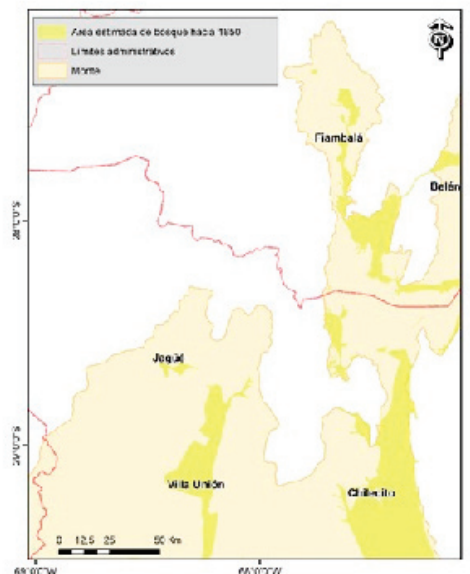

Distribución actual

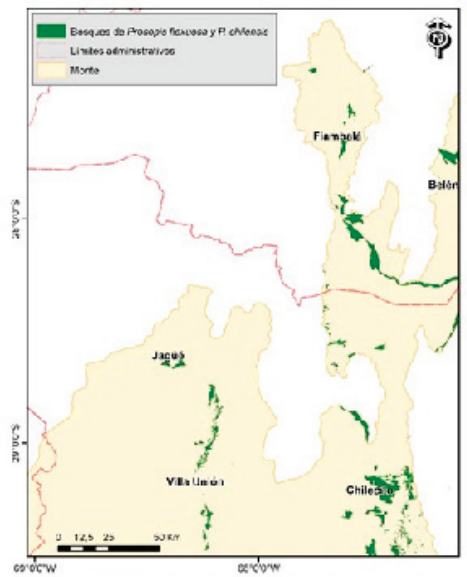

Figura 4. Comparación entre la distribución potencial, indicada a través de la probabilidad de ocurrencia bosques (Perosa et al. 2014), la distribución en 1850 según fuentes documentales (Rojas et al. 2014b) y distribución actual en la zona septentrional del Monte (Rojas 2013; Alvarez et al. 2015). Se puede observar cómo las áreas con mayor probabilidad de ocurrencia de bosque tuvieron bosques en tiempos históricos y mantienen bosque actualmente. También las áreas donde se retrajo la distribución del bosque durante los últimos 150 años. La ubicación relativa del área mostrada se puede observar en la Figura 1. Mapas: F. Rojas.

Figure 4. Comparison between potential distribution, indicated by the probability of woodland occurrence (Perosa et al. 2014), distribution in 1850 according to documentary sources (Rojas et al. 2014b) and current distribution (Rojas 2013; Alvarez et al. 2015). It shows how areas with the highest woodland occurrence probability had forests in historical times and maintain woodlands today. In addition, it shows the areas where woodland distribution declined over the past 150 years. The relative location of the displayed area can be seen in Figure 1. Mapping: F. Rojas. 
reforestadas, características productivas y aspectos concernientes a la conservación de la biodiversidad en estos ambientes frágiles (FAO 2016). Por otro lado, el desarrollo incipiente de planes de ordenamiento forestal se explica por la llegada escasa de los datos generados en los programas científicos a los gestores del bosque y a sus habitantes. Queda pendiente el trabajo de profundizar la interpretación y transferencia de los datos existentes y generar los conocimientos faltantes.

La sustentabilidad en sentido estricto puede no ser posible a escala local, al menos en las zonas más áridas del Monte, pero sí es posible contribuir a la sustentabilidad de los manejos al optimizar variables ambientales, sin disminuir las socioeconómicas o viceversa. Goiran et al. (2013) sugieren que existe una relación entre la capacidad de proveer recursos y servicios, y la distribución de puestos ganaderos y las actividades humanas. Sin embargo, factores socioeconómicos como el uso de la tierra, los cambios políticos y sociales, las mejoras en infraestructura y servicios, la apertura de mercados, entre otros, pueden inducir modificaciones en la forma y la distribución del manejo del bosque. Estos cambios pueden generar mejoras en la calidad de vida a escala local y en el corto plazo, pero también promover a largo plazo una pérdida de sustentabilidad de los sistemas cuando no se tiene en cuenta el eje ambiental, incluso llevando al posterior abandono de la tierra.

El gran desafío es poder realizar un ordenamiento territorial que considere el potencial productivo de cada zona en función de los limitantes ambientales, que mejore los procesos tecnológicos usados, a fin de optimizar el aprovechamiento y que permita decidir las actividades prioritarias para cada área. La Ley 26331 de Presupuestos Mínimos de Protección Ambiental de los Bosques Nativos le ordena a cada provincia la realización de un Ordenamiento Territorial de Bosques Nativos (OTBN) orientado al ordenamiento forestal sustentable. Estos OTBN y los planes de manejo y conservación asociados representan una herramienta para configurar los escenarios de posibles usos de los productos del bosque. El panorama de esta temática en el mediano plazo es complejo. Cada una de las jurisdicciones (i.e., provincias) definió oportunamente su OTBN y priorizó diferentes aspectos. En algunos casos, estas diferencias responden a las particularidades ambientales y al uso histórico, y, en otros, a priorizaciones políticas; por lo tanto, la aplicación de los OTBN en cada jurisdicción posiblemente muestre resultados diferentes ante condiciones similares.

Aunque el efecto de la aplicación de la ley todavía es incipiente, se dio un primer paso en determinar espacialmente los posibles tipos de manejo. Se está trabajando para unificar técnicas de monitoreo a largo plazo, que representan un insumo básico para aunar criterios a escala regional o de cuenca, más allá de los límites jurisdiccionales. Lo discutido en esteartículo sugiere que es necesarioincorporar a los OTBN políticas territoriales con una visión a largo plazo que incluya la heterogeneidad espacial de las condiciones ambientales y la recuperación del bosque y de la provisión de servicios ecosistémicos, en conjunto con las demandas socioeconómicas y el bienestar de los pobladores locales. Es importante resaltar que entre las posibles actividades no sólo se debe contemplar la planificación del uso forestal, sino la integración de otras actividades, principalmente la ganadería, y necesidades como la conservación pura o la restauración ecológica en los casos en los que la recuperación natural no funcione.

Agradecimientos. A F. Rojas por la realización de los mapas y a M. Cony, E. Cesca, P. Meglioli, C. Vega Riveros y F. Rojas por la discusión de las ideas. Los trabajos mencionados en este artículo fueron financiados por CONICET, FONCyT, UNCUYO, UCAR, SAyDS de la Nación.

\section{REFERENCIAS}

Abraham, E. M., and M. R. Prieto. 1999. Vitivinicultura y desertificación en Mendoza. Pp. 109-135 en B. García Martínez (ed.). Estudios de historia y ambiente en América: Argentina, Bolivia, México, Paraguay. IPGH - Colegio de México, México.

Allegretti, L. I., C. B. Passera, J. Páez, A. Úbeda, C. Sartor, and A. B. Robles. 2005. Capacidad sustentadora y composición botánica de la ingesta caprina en un ecosistema árido, Lavalle, Argentina. Pp. 221-228 en K. Osoro Otadui et al. (eds.). Producciones agroganaderas: Gestión eficiente y conservación del medio natural, España.

Alvarez, J. A., and P. E. Villagra. 2009. Prosopis flexuosa DC. (Fabaceae, Mimosoideae). Kurtziana 35:49-63.

Alvarez, J. A., P. E. Villagra, E. M. Cesca, F. Rojas, and S. Delgado. 2015. Estructura, distribución y estado de conservación de los bosques de Prosopis flexuosa del Bolsón de Fiambalá (Catamarca). Boletín de la Sociedad Argentina de Botánica 50:193-208. 
Alvarez, J. A., P. E. Villagra, M. A. Cony, E. Cesca, and J. A. Boninsegna. 2006. Estructura y estado de conservación de los bosques de Prosopis flexuosa D.C. en el Noreste de Mendoza, Argentina. Revista Chilena de Historia Natural 79:75-87.

Alvarez, J. A., P. E. Villagra, B. E. Rossi, and E. Cesca. 2009. Spatial and temporal litterfall heterogeneity generated by woody species in the Central Monte desert. Plant Ecology 205:295-303.

Bastin, J., N. Berrahmouni, A. Grainger, D. Maniatis, D. Mollicone, et al. 2017. The extent of forest in dryland biomes. Science 638:635-638.

Cesca, E. M., P. E. Villagra, and J. A. Alvarez. 2014. From forest to shrubland: Structural responses to different fire histories in Prosopis flexuosa woodland from the Central Monte (Argentina). Journal of Arid Environments 110:1-7.

Cesca, E. M., P. E. Villagra, C. B. Passera, and J. A. Alvarez. 2012. Effect of Prosopis flexuosa on understory species and its importance to pastoral management in woodlands of the Central Monte Desert. Revista de la Facultad de Ciencias Agrarias 44:211-223.

Contreras, L. C., E. Jobbágy, P. E. Villagra, M. D. Nosetto, and J. Puigdefábregas. 2011. Remote sensing estimates of supplementary water consumption by arid ecosystems of Central Argentina. Journal of Hidrology 397:10-22.

Cony, M. A. 1996. Genetic variability in Prosopis flexuosa D. C., a native tree of the Monte phytogeographic province, Argentina. Forest Ecology and Management 87:41-49.

Cuba Salerno, A. B. 1998. Desarrollo rural sostenible en los bosques secos de la Costa Norte del Perú: el Proyecto Algarrobo. Pp. 41-61 en A. B. Cuba Salerno et al. (eds). Bosques secos y desertificación. INRENA - Proyecto Algarrobo, Lambayeque, Perú.

FAO 2007. Situación de los bosques del mundo. FAO, Roma, Italia

FAO 2016. Documento sobre el proceso de FRA 2015. Documento de Trabajo de la Evaluación de los Recursos Forestales No. 186. FAO. Roma. Italia

Goirán, S. B., A. Tonolli, J. N. Aranibar, P. E. Villlagra, E. Millan, L. Forconesi, and E. M. Bringa. 2013. Factores que determinan el uso del espacio y los recursos en zonas áridas no irrigadas del NE de Mendoza (Argentina). Pp. 97109 en A. Lara et al. (eds). Servicios ecosistémicos hídricos: estudios de caso en América Latina y el Caribe. Valdivia, Chile. Red ProAgua CYTED, Imprenta América., Valdivia, Chile.

Guevara, J. C., E. Grünwaldt, O. R. Estevez, A. J. Bisigato, L. J. Blanco, F. N. Biurrun, C. A. Ferrando, C. C. Chirino, E. F. Morici, B. Fernández, L. I. Allegretti, and C. B. Passera. 2009. Range and livestock production in the Monte Desert, Argentina. Journal of Arid Environments 73:228-237.

Jobbágy, E. G., M. D. Nosetto, P. E. Villagra, and R. B. Jackson. 2011. Water subsidies from mountains to deserts: Their role sustaining groundwater-fed oases in a sandy landscape. Ecological Applications 21:678-694.

Karlin, U., and R. Díaz. 1984. Potencialidad y Manejo de Algarrobos en el Arido Subtropical Argentino. Secretaría de Ciencia y Técnica, República Argentina.

Labraga, J. C., and R. Villalba. 2009. Climate in the Monte Desert: past trends, present conditions, and future projections. Journal of Arid Environments 73:154-163.

Martínez de Escobar, S., P. Neira, and P. Lucero. 2015. Algarroba. Alimento ancestral. Grupo de Estudios de Naturaleza y Cultura en el Árido, Universidad Nacional de San Juan, Argentina.

Meglioli, P. A., J. N. Aranibar, P. E. Villagra, J. A. Alvarez, and E. G. Jobbágy. 2014. Livestock stations as foci of groundwater recharge and nitrate leaching in a sandy desert of the Central Monte, Argentina. Ecohydrology 7: 600-611.

Miles, L., A. C. Newton, R. S. DeFries, C. Ravilious, I. May, S. Blyth, V. Kapos, and J. E. Gordon. 2006. A global overview of the conservation status of tropical dry forests. Journal of Biogeography 33:491-505.

Morello, J. 1958. La Provincia Fitogeográfica del Monte. Opera Lilloana 2:5-115.

Perosa, M., J. F. Rojas, P. E. Villagra, M. F. Tognelli, R. Carrara, and J. A. Alvarez. 2014. Distribución potencial de los bosques de Prosopis flexuosa en la Provincia Biogeográfica del Monte (Argentina). Ecología Austral 24:238-248.

Prieto, M. R., and E. M. Abraham. 1998. Historia ambiental del sur de Mendoza (Siglos XVI al XIX). Los factores críticos de un Proceso de Desertificación. Bamberger Geographics Schriften Bd. 15:277-297.

Prieto, M. R., P. E. Villagra, N. B. Lana, and E. M. Abraham. 2003. Utilización de documentos históricos en la reconstrucción de la vegetación de la Llanura de la Travesía (Argentina) a principios del siglo XIX. Revista Chilena de Historia Natural 76:613-622.

Roig, F. A. 1993. Aportes a la etnobotánica del género Prosopis. Pp. 99-119 en IADIZA (ed.). Conservación y mejoramiento de especies del género Prosopis. CIID - IADIZA, Mendoza, Argentina.

Rojas, F. 2013. Rol de la minería y el ferrocarril en el desmonte del oeste riojano y catamarqueño (Argentina) en el período 1851-1942. Población y Sociedad 20:5-39.

Rojas, J. F., M. D. R. Prieto, P. E. Villagra, and J. A. Alvarez. 2014a. Deforestación y actividades productivas en los valles del oeste de La Rioja y Catamarca, desde mediados del siglo XIX hasta la actualidad. Boletín de Estudios Geográficos 103:19-57.

Rojas, J. F., M. R. Prieto, P. E. Villagra, and J. A. Alvarez. 2014b. Distribución espacial de los bosques nativos en el Norte del Monte argentino, hacia mediados del siglo XIX. Historia 2.0 4:31-46.

Rossi, B. E., and P. E. Villagra. 2003. Effects of Prosopis flexuosa on soil properties and the spatial pattern of understory species in arid Argentina. Journal of Vegetation Science 14:543-550.

Sarandón, S. J. 2002. El desarrollo y uso de indicadores para evaluar la sustentabilidad de los agroecosistemas. Pp. 
393-447 en S. J. Sarandón, (ed.). Agroecología: El Camino hacia una Agricultura Sustentable. Ediciones Cientificas Americanas E.C.A., La Plata, Argentina.

Solbrig, O. T., and P. D. Cantino. 1975. Reproductive adaptations in Prosopis (Leguminosae, Mimosoideae). Journal of the Arnold Arboretum 56:185-210.

Vilela, A., M. L. Bolkovic, P. Carmanchahi, M. Cony, D. de Lamo, and D. Wassner. 2009. Past, present and potential uses of native flora and wildlife of the Monte Desert. Journal of Arid Environments 73:238-243.

Villagra, P. E., J. A. Boninsegna, J. A. Alvarez, M. Cony, E. Cesca, and R. Villalba. 2005. Dendroecology of Prosopis flexuosa woodlands in the Monte desert: Implications for their management. Dendrochronologia 22:209-213.

Villagra, P. E., M. A. Cony, N. G. Mantován, B. E. Rossi, M. M. González Loyarte, R. Villalba, and L. Marone. 2004. Ecología y manejo de los algarrobales de la Provincia Fitogeográfica del Monte. En M. F. Arturi et al. (eds.). Ecología y Manejo de Bosques Nativos de Argentina. Editorial Universidad Nacional de La Plata, Buenos Aires, Argentina.

Villagra, P. E., G. E. Defossé, H. F. Del Valle, M. S. Tabeni, C. M. Rostagno, E. Cesca, E. M. Abraham, S. Tabeni, and M. Rostagno. 2009. Land use and disturbance effects on the dynamics of natural ecosystems of the Monte Desert. Implications for their management. Journal of Arid Environments 73:202-211.

Villagra, P. E., C. B. Passera, S. Greco, C. Sartor, J. N. Aranibar, P. A. Meglioli, J. A. Alvarez, L. Allegretti, M. E. Fernández, M. A. Cony, P. C. Kozub, and C. Vega Riveros. 2017. Uso de plantas nativas en la restauración y recuperación productiva de ambientes salinos de las zonas áridas de la región del Monte, Argentina. Pp. 419-444 en E. Taleisnik and R. S. Lavado (eds.). Ambientes salinos y alcalinos de la Argentina. Universidad Católica de Córdoba - Orientación Gráfica Editora, Córdoba, Argentina. 